\title{
THYROID DYSFUNCTION AND BLOOD PRESSURE COMPONENTS: MULTIVARIATE STATISTICAL ASSOCIATIONS
}

\section{MEENAKSHISUNDARAM R. ${ }^{1,2^{*}}$, GROOTVELD M. ${ }^{2}$, NIRUBHANBHARATHY R.V. ${ }^{3}$, NITHYA E. ${ }^{3}$, MAJELLA L.J. ${ }^{3}$ AND THIRUMALAIKOLUNDUSUBRAMANIAN P.1}

${ }^{1}$ Chennai Medical College Hospital and Research Centre, Irungalur, Trichy- 621 105, TN, India.

2Leicester School of Pharmacy, Faculty of Health and Life Sciences, De Montfort University, The Gateway, Leicester, LE1 9BH, UK.

${ }_{3}^{3}$ Madurai Medical College, Madurai- 625 020, TN, India.

*Corresponding Author: Email- rmsundarchandran@gmail.com

Received: March 05, 2014; Accepted: June 23, 2014

\begin{abstract}
-
Background: Although thyroid dysfunctions exert significant effects on blood pressure (BP), published literature available has revealed contradictory data.

Objectives: To explore the inter-relationships between selected thyroid dysfunctional status (hyper and hypothyroid conditions) and established biomarkers (thyroid stimulating hormone (TSH) and thyroxine (T4)); and BP components (specifically Systolic BP (SBP), Diastolic BP (DBP), and Mean Arterial Pressure (MAP), and uniquely SBP:DBP ratio).

Materials and Methods: We followed rigid criteria in order to select adults with hyperthyroidism $(n=71)$ and hypothyroidism ( $n=300)$, together with healthy age-matched controls ( $n=300$ ), and applied a series of statistical analysis strategies to the datasets acquired.

Results: Univariate statistical analysis strategies of the SBP:DBP ratio by ANOVA and ANCOVA revealed that its mean value was significantly greater in hyperthyroid patients than those of healthy control and hypothyroid subjects $(p<0.0001)$. Body mass index (BMI) was significantly elevated in hypothyroid patients over those of healthy control and hyperthyroid subjects $(p<0.0001)$; healthy controls had a significantly greater BMI value than that of hyperthyroid patients $(p<0.0001)$. Multivariate (MV) analysis [redundancy analysis (RDA), canonical correlation analysis (CCorA), and principal component analysis (PCA)] demonstrated powerful relationships between all blood pressure components and thyroid disease classifications, together with their biomarker indices and further patient-related parameters.

Conclusions: Since thyroid dysfunctional status is associated with elevated BP, and increasing BP is positively-correlated with elevated serum thyroid biomarkers, hyper and hypothyroid disorders should be recognized and treated early in order to avoid critical hazards presented by high BP.
\end{abstract}

Keywords- Hyperthyroidism, hypothyroidism, thyroid biomarkers, thyroxine, thyroid stimulating hormone, SBP, DBP, MAP, SBP:DBP ratio, body mass index, blood pressure, hypertension

Citation: Meenakshisundaram R., et al. (2014) Thyroid Dysfunction and Blood Pressure Components: Multivariate Statistical Associations. International Journal of Medical and Clinical Research, ISSN: 0976-5530 \& E-ISSN: 0976-5549, Volume 5, Issue 1, pp.-294-304.

Copyright: Copyright@2014 Meenakshisundaram R., et al. This is an open-access article distributed under the terms of the Creative Commons Attribution License, which permits unrestricted use, distribution and reproduction in any medium, provided the original author and source are credited.

\section{Introduction}

Thyroid hormones exert a range of actions on physiological systems of the body, including cardiovascular physiology. Thyroid dysfunction is associated with increased mortality from, or a high prevalence of, cardiovascular diseases [1]; deviations from euthyroid status affect normal physiology. Although the effect of such deviations on blood pressure (BP) is more marked [2,3], the relationship between thyroid disorders and BP is not well recognized [4]. Moreover, to date, a series of contradictory relationships have been noted. Indeed, a linear relationship between serum TSH levels and BP (both systolic and diastolic) was observed by Asvold, et al $[5,6]$. However, no such associations were observed in two further stud- ies $[7,8]$. Physiologically, BP is maintained via cardiac output (CO) and systemic vascular resistance (SVR), and hence BP is calculated using the CO x SVR formula. Through multiple mechanisms, thyroid hormones maintain heart rate, cardiac contractility and blood volume, and hence exert an important influence on BP. In addition, thyroid hormones regulate BP through the renin-angiotensinaldosterone system (RAAS), in which hyper and hypothyroidism induce opposite effects [4]. Moreover, thyroid disease can cause endothelial dysfunction through reduced fibrinolytic activity and nitric oxide (NO) synthesis [1]. At the molecular level, thyroid hormones play an important role in the gene expression of cardiac myocytes [9]. Furthermore, hypothyroidism has been associated 
with one or other metabolic syndrome components such as hyperlipidemia, diabetes and obesity [10]

In this investigation, we have explored inter-relationships between thyroid dysfunctional status, together with their associated biomarkers and a range of $\mathrm{BP}$ components [specifically systolic BP (SBP), diastolic BP (DBP), Mean Arterial Pressure (MAP), and uniquely SBP:DBP ratio] monitored in a very large number of healthy control, hypothyroid and hyperthyroid patients. To the best of our knowledge, to date this study represents the largest one available which describes and explores the complex interrelationships between thyroid disease status and a series of $\mathrm{BP}$ monitoring criteria.

\section{Materials and Methods}

This study was performed at a tertiary care center in Southern India after the acquisition of approval from the Institutional research Ethics Committee, and also on receipt of informed consent from each participant recruited to the investigation. The ethical clearnace approval was obtained from Research Ethics Committee, Chennai Medical College Hospital \& Research Centre, Trichy, India.

\section{Subject Criteria}

Rigid inclusion and exclusion criteria were applied to recruit adult participants with thyroid dysfunctional status (established hypothyroid and hyperthyroid cases) and healthy control (euthyroid) subjects. Subjects with co-existing morbidities such as diabetes, postthyroidectomy status, cardiovascular diseases, renal dysfunction and other illnesses were excluded from the study. Also, all the subjects in our study were non-smokers (never smoked), non-alcoholic and all of them consumed normal salt intake. There were 71 hyperthyroid and 300 hypothyroid cases, and 300 control subjects. Anthropometric (height and weight) and BP components were determined for all these participants. For the purpose of this study, the $\mathrm{BP}$ components considered are systolic BP (SBP), diastolic BP (DBP), mean arterial pressure (MAP), and SBP:DBP ratio. MAP values were computed using the [DBP $+1 / 3$ (pulse pressure)] formula, where pulse pressure represents the difference between the SBP and DBP values. Body mass index (BMI) was calculated using the formula weight in $\mathrm{kg} /$ (height in meters) ${ }^{2}$. $\mathrm{A} \mathrm{BMl}$ value 23.0 and above was considered as overweight according to the WHO for Indians [11].

\section{Thyroid Function Evaluation}

Thyroid-stimulating hormone (TSH) and free thyroxine (T4) were estimated in all subjects by immunoradiometric assay (IRMA) using kits procured from Board of Radiation and Isotope Technology (BRIT), Mumbai, India. Normal reference population values of TSH and T4 are $0.17-4.05 \mathrm{IU} / \mathrm{ml}$ and $55-135 \mathrm{ng} / \mathrm{ml}$ respectively.

\section{Statistical Analysis of Experimental Data Acquired}

Univariate data analysis of SBP, DBP, SBP:DBP ratio, MAP, BMI, age, and blood serum TSH and T4 level indices [analysis of variance (ANOVA), analysis of covariance (ANCOVA) and $X^{2}$ contingency table analysis] was performed using XLSTAT2013 software. ANOVA and ANCOVA were conducted both with and without the inclusion of first-order interaction variance components, and also with a tolerance of 0.0001 and the computation of $95 \%$ confidence intervals (Cls) for the least squares means determined. In cases where there were heterogeneous intra-sample variances, comparisons of mean thyroid status values were performed using the Welch
ANOVA method. Post-hoc analysis of the differences between the mean values of all group classifications was performed by Tukey's honestly significantly difference (HSD), Fisher's least significant difference (LSD), and the Bonferroni and Dunn-Sidak tests.

Partial correlation coefficients [and their corresponding two-tailed significance $(p)$ values] were computed between a MV dataset containing the serum T4 and TSH biomarker concentrations (together with age and BMI values) and each of the BP components individually.

Multivariate Redundancy Analysis (RDA) was also performed with XLSTAT2013 software, the model involving a minimum filter factor of $80 \%$; permutations were performed $500,1,000$ or 2,000 times, and both response (BP) and explanatory (thyroid disease) variables were centered and reduced prior to analysis (a 1\% significance level was employed), and weighted average (WA) scores were employed for the response variables. For partial RDA, a preliminary stage was performed in which the explanatory variables (thyroid status, both with and without serum T4 and TSH biomarker concentrations and/or BMI, age and gender) table was sub-divided into two groups, the first of which $[X(1)]$ contained conditioning variables (BMI, age and gender), the effect of which was removed, since their effects on BP components are already known). Regressions were then performed on the $Y$ (blood pressure) and $X(2)$ (thyroid status, both with and without serum TSH and T4 levels) tables, and the residuals of the regressions arising were then employed for the RDA step. In this manner, we analysed the effects of the second $[X$ (2)] group of variables, i.e. that remaining after the effect of the first [X(1)] group of conditioning variables had been removed.

Canonical correlation analysis (CCorA) was also performed using XLSTAT2013 software and a minimum filter factor of $80 \%$, as was principal component analysis (PCA). PCA was conducted both with and without a subsequent Varimax rotation (the latter with Kaiser normalisation and a maximum of 4 components), and the application of Bartlett's sphericity test. For the PCA performed on the BP index dataset reported below, variables were standardised (meancentered and then divided by the sample variance) before conducting the analysis.

The CCorA model developed here involved the analysis of the score vectors derived from pre-isolated orthogonal Principal Components (PCs) arising from the PCA of two separate datasets, the first from one which incorporated the blood serum thyroid biomarker (T4 and TSH) levels, an assigned thyroid disease status score, age, $\mathrm{BMI}$, and also a gender score index, the second from the series of correlated SBP, DBP, MAP and SBP:DBP ratio parameters. Datasets were cubed root-transformed and Pareto-scaled prior to the performance of the primary PCA stage of the overall canonical correlation analysis.

\section{Results \\ Descriptive Statistics}

The number of males and females, and their overall mean age for hyperthyroid, hypothyroid and euthyroid control patients were 19 and 59 , and 35.59 yr.; 17 and 81 , and 34.15 yr.; and 18 and 86 , and $38.40 \mathrm{yr}$.; respectively. The range and mean values of BP components for hyperthyroid, hypothyroid and euthyroid control patients were SBP: 108 to 150 , and $127.04 \mathrm{mmHg} ; 80$ to 200 , and 120.31 $\mathrm{mmHg} ; 90$ to 160 , and $115.04 \mathrm{mmHg}$; respectively. DBP: 48 to 92 , and $77.80 \mathrm{mmHg} ; 60$ to 140 , and $80.59 \mathrm{mmHg} ; 70$ to 100 , and $77.28 \mathrm{mmHg}$ respectively; MAP: 68.67 to 110.00 , and $94.04 \mathrm{mmHg}$; 
73.33 to 160.00 , and $93.83 \mathrm{mmHg} ; 76.67$ to 120 , and $89.87 \mathrm{mmHg}$ respectively. SBP:DBP ratio: 1.3750 to 2.2917 , and $1.637 ; 1.1429$ to 1.8333 , and $1.495 ; 1.1250$ to 1.8286 , and 1.492 respectively.

Similarly, the range and mean BMI values for hyperthyroid, hypothyroid and euthyroid control patients were 14 to 21 , and 17.71 kg.m-2; 13 to 33 , and $22.39 \mathrm{~kg} \cdot \mathrm{m}^{-2} ; 16$ to 24 , and $21.38 \mathrm{~kg} \cdot \mathrm{m}^{-2}$ respectively. The range and mean values of serum thyroid disease biomarkers for hyperthyroid, hypothyroid and control euthyroid patients were TSH: 0 to 0.13 , and $0.085 \mathrm{IU} / \mathrm{ml} ; 26.00$ to 100.00 , and $75.00 \mathrm{IU} / \mathrm{ml} ; 0.10$ to 5.10 , and $2.07 \mathrm{IU} / \mathrm{ml}$ respectively. $\mathrm{T} 4: 160.00$ to 240.00 , and $198.52 \mathrm{ng} / \mathrm{ml} ; 50.00$ to 113.00 , and $65.53 \mathrm{ng} / \mathrm{ml} ; 55.00$ to 147.00 , and $96.34 \mathrm{ng} / \mathrm{ml}$ respectively.

\section{Univariate Statistical Analysis}

ANOVA of the dataset revealed very highly significant differences between the disease classifications for (1) SBP, the mean values

[Fig-1](a) being in the order Hyperthyroid >> Hypothyroid > Euthyroid $(p<0.0001)$; (2) DBP, the mean value [Fig-1](b) order being Hypothyroid > Hyperthyroid $\approx$ Euthyroid $(p<0.0001)$; (3) SBP:DBP ratio, the mean value [Fig-1](c) order being Hyperthyroid $\gg>$ Hypothyroid $\approx$ Euthyroid $(p<0.0001)$; (4) MAP, the mean value [Fig-1](d) order being Hyperthyroid $\approx$ Hypothyroid $>$ Euthyroid $(p<0.0001)$; and finally (5) BMl, the mean value [Fig-1](e) order being Hypothyroid > Euthyroid $>>$ Hyperthyroid $(p<0.0001)$. The mean serum TSH [Fig-1](f) and T4 [Fig-1](g) levels were in the order hypothyroid $>$ euthyroid $>>$ hyperthyroid $(p<0.0001)$, and hyperthyroid $>>$ euthyroid $\gg$ hypothyroid $(p<0.0001)$ respectively [Table-1]. As expected, analysis of the untransformed datasets revealed that there were also highly significant differences between the variances ('scatter') of these BP components between thyroid status groups, for example Hypothyroid $\approx$ Hyperthyroid $\gg$ Euthyroid for SBP $(p<0.0001)$; Hypothyroid $>>$ Hyperthyroid > Euthyroid for DBP $(p<0.0001)$; Hyperthyroid $>>$ Hypothyroid $\approx$ Euthyroid for SBP:DBP ratios $(p=0.005)$; and Hypothyroid $>>$ Hyperthyroid $>$ Euthyroid for $\operatorname{MAP}(p<0.0001)$. Interestingly, the variance of the BMI values of the hypothyroid patient group was found to be much greater than those of both the euthyroid and hyperthyroid patient groups $(p<0.0001)$.

Focusing on the SBP:DBP ratio in view of the clear, highly significant differences in this parameter between the disease classification groups, further univariate analysis was performed. An ANCOVA model without allowing for any interaction effects demonstrated that there were extremely highly significant differences between disease classifications $(p<0.0024)$, again predominantly ascribable to the much higher SBP:DBP ratio of hyperthyroid patients than those of hypothyroid and euthyroid status, and also that serum T4 and TSH concentrations exerted highly significant effects as covariates $(p<0.0001$ and 0.00572 respectively) on this BP component; all further (explanatory) covariates, i.e. BMI values, age and gender, were found to not exert a significant influence on this (dependent) SBP:DBP ratio variable. Standardised regression coefficients (and the sign of these contributions) for each of the significant covariates in this model were in the order hyperthyroid status $(+)>$ euthyroid status $(-)>$ hypothyroid status $(-)>$ serum TSH level $(-)>$ serum T4 level $(+)$, i.e. there were positive and negative contributions of serum T4 and TSH concentrations towards the SBP:DBP ratio dependent variable, as expected.

ANCOVA performed with allowance for all possible first-order interaction effects demonstrated that the serum TSH level-thyroid status interaction component of variance was extremely significant $(p<0.0001)$, with also a significant effect for the serum T4-TSH concentrations interaction effect $(p=0.01024)$.

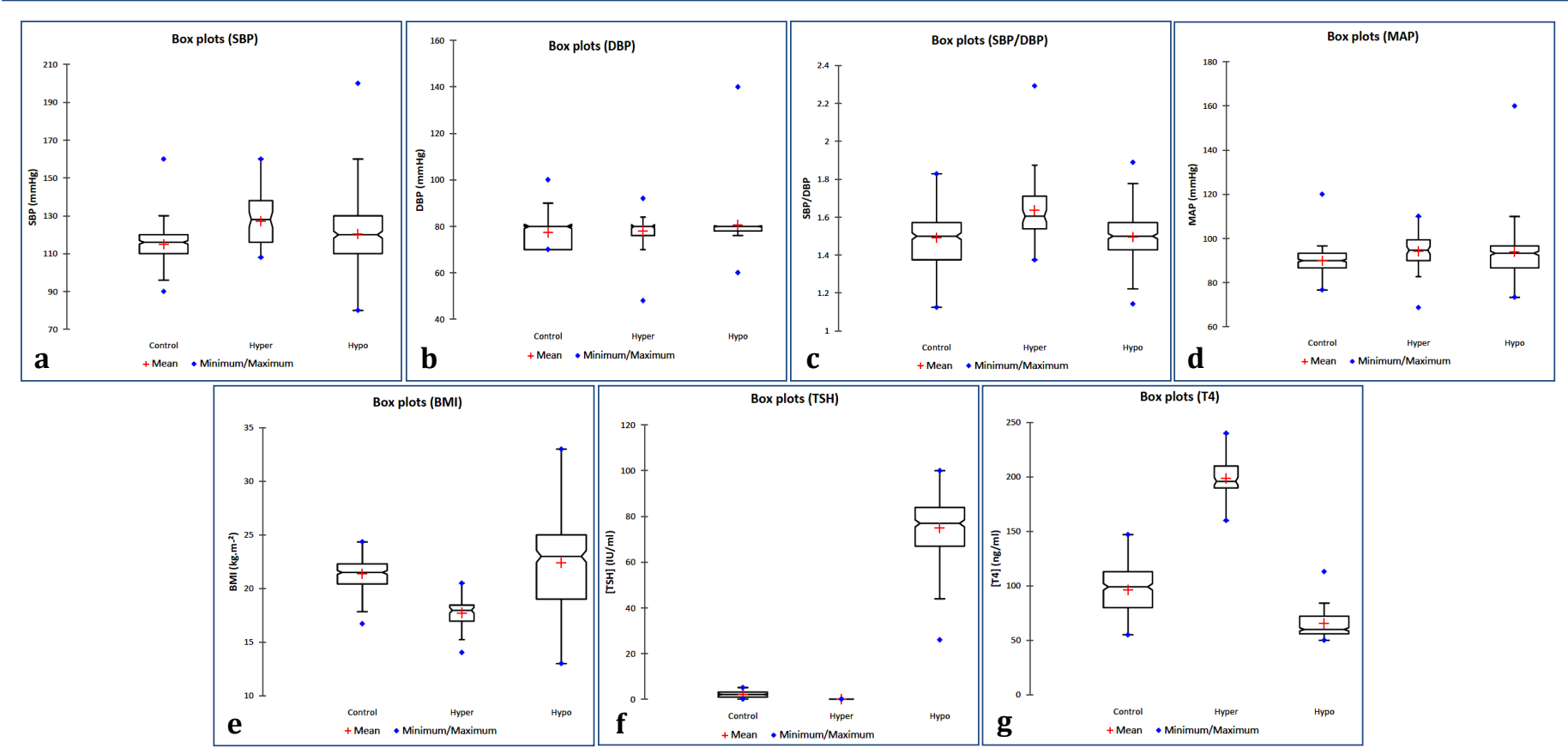

Fig. 1- Notched box and whisker diagrams of (a) SBP, (b) DBP, (c) SBP:DBP ratio, (d) MAP, (e) BMI, (f) blood serum TSH level and (g) blood serum T4 level for each thyroid status classification. The mean value of each dataset is shown as a red cross, and the median is visible as the horizontal line within the boxes. The minimum and maximum values are indicated by blue rhombuses, and the box and notch limits correspond to the first or third dataset quartiles, and $95 \%$ confidence intervals $(\mathrm{Cls})$ for the median values, respectively. The lower 'whiskas' limit is given by Q1 - [1.50 (Q3-Q1)], where Q1 and Q3 denote the first and third quartiles, respectively, and the upper one by Q3 + [1.50(Q3-Q1)]. The width of the boxes is proportional to the square root of the sample size (n). 
Table 1- Mean $\pm 95 \%$ confidence intervals (Cls) for SBP, DBP, SBP:DBP ratio, MAP, BMI, T4 and TSH level, and Age parameters determined for Hypothyroid $(n=300)$, Hyperthyroid $(n=71)$ and Euthyroid $(n=300)$ patients. The significance of differences between the mean values of the three thyroid status classifications were determined by ANOVA.

\begin{tabular}{|c|c|c|c|c|c|}
\hline Parameter & Thyroid Status & Mean $\pm 95 \%$ Cls & $\begin{array}{c}\text { p Value Euthyroid vs. } \\
\text { Hypothyroid }\end{array}$ & $\begin{array}{c}\text { p Value Euthyroid vs. } \\
\text { Hyperthyroid }\end{array}$ & $\begin{array}{c}\text { p Value Hypothyroid vs. } \\
\text { Hyperthyroid }\end{array}$ \\
\hline & Hypothyroid & $120.31 \pm 1.79$ & & & \\
\hline \multirow[t]{3}{*}{ SBP $(m m H g)$} & Hyperthyroid & $127.04 \pm 3.30$ & $<0.0001$ & $<0.0001$ & 0.00016 \\
\hline & Euthyroid & $115.04 \pm 1.13$ & & & \\
\hline & Hypothyroid & $80.59 \pm 1.00$ & & & \\
\hline \multirow[t]{3}{*}{$\mathrm{DBP}(\mathrm{mmHg})$} & Hyperthyroid & $77.80 \pm 1.46$ & $<0.0001$ & ns & 0.002 \\
\hline & Euthyroid & $77.28 \pm 0.59$ & & & \\
\hline & Hypothyroid & $1.495 \pm 0.014$ & & & \\
\hline \multirow[t]{3}{*}{ SBP:DBP Ratio } & Hyperthyroid & $1.637 \pm 0.040$ & ns & $<0.0001$ & $<0.0001$ \\
\hline & Euthyroid & $1.492 \pm 0.015$ & & & \\
\hline & Hypothyroid & $93.83 \pm 1.19$ & & & \\
\hline \multirow[t]{3}{*}{ MAP (mmHg) } & Hyperthyroid & $94.04 \pm 1.77$ & $<0.0001$ & $<0.0001$ & ns \\
\hline & Euthyroid & $89.87 \pm 0.65$ & & & \\
\hline & Hypothyroid & $22.39 \pm 0.42$ & & & \\
\hline \multirow[t]{3}{*}{ BMI $\left(\mathrm{kg} \cdot \mathrm{m}^{-2}\right)$} & Hyperthyroid & $17.71 \pm 0.32$ & $<0.0001$ & $<0.0001$ & $<0.0001$ \\
\hline & Euthyroid & $21.38 \pm 0.16$ & & & \\
\hline & Hypothyroid & $65.53 \pm 1.32$ & & & \\
\hline \multirow[t]{3}{*}{ Serum T4 level (ng/ml) } & Hyperthyroid & $198.52 \pm 4.04$ & $<0.0001$ & $<0.0001$ & $<0.0001$ \\
\hline & Euthyroid & $96.34 \pm 2.54$ & & & \\
\hline & Hypothyroid & $75.00 \pm 1.94$ & & & \\
\hline \multirow[t]{3}{*}{ Serum TSH level (IU/ml) } & Hyperthyroid & $0.085 \pm 0.008$ & $<0.0001$ & $<0.0001$ & $<0.0001$ \\
\hline & Euthyroid & $2.073 \pm 0.164$ & & & \\
\hline & Hypothyroid & $34.15 \pm 1.37$ & & & \\
\hline \multirow[t]{2}{*}{ Age (yr.) } & Hyperthyroid & $35.59 \pm 2.75$ & 0.002 & ns & ns \\
\hline & Euthyroid & $38.36 \pm 1.54$ & & & \\
\hline
\end{tabular}

ns: not significant.
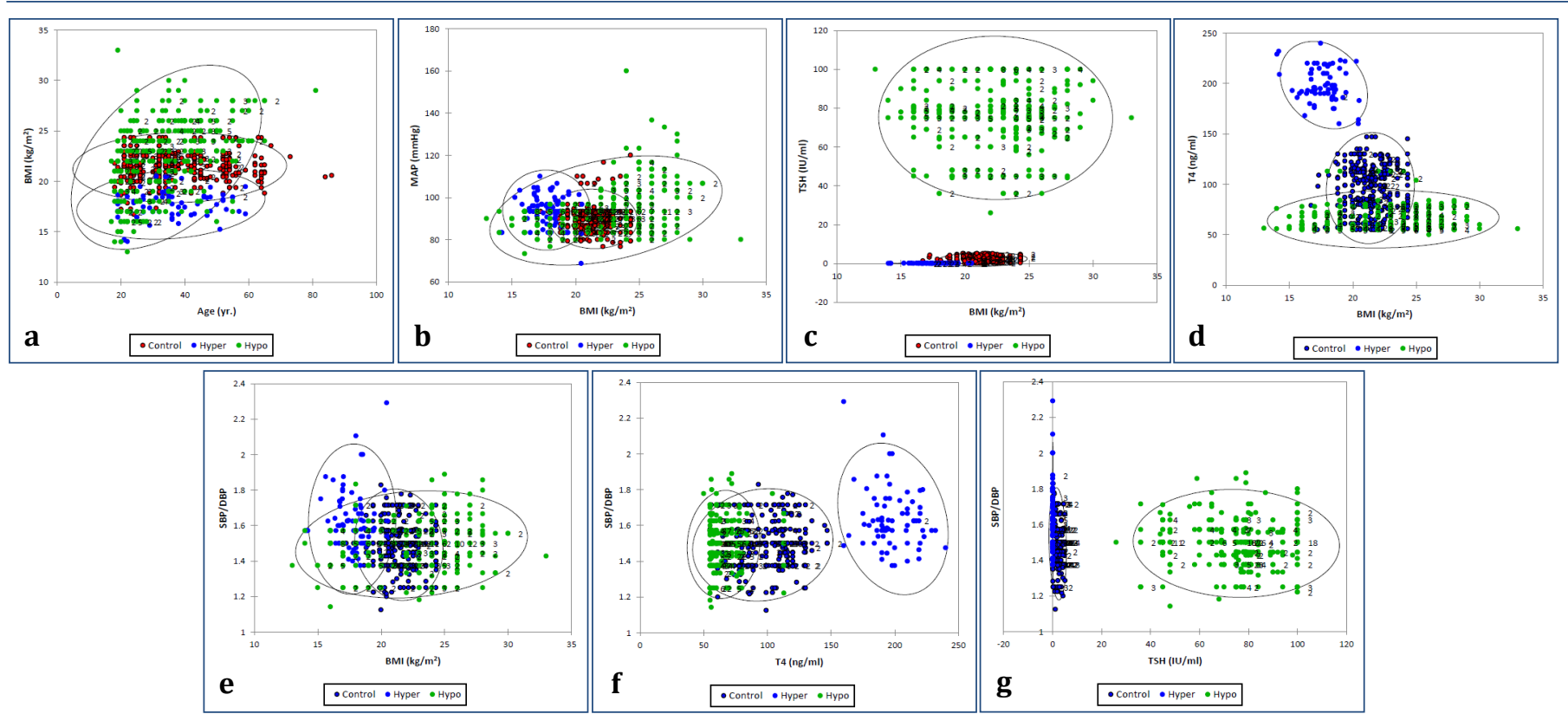

Fig. 2- Plots of (a) BMI versus Age; (b), (c) and (d), MAP value, blood serum TSH level and blood serum T4 level, respectively, versus BMI for each thyroid status group (hypothyroid, hyperthyroid and euthyroid); (e), (f) and (g) show plots of SBP:DBP ratio versus BMI, serum T4 level and serum TSH level respectively. The non-scalar numbers indicate frequencies $>1$ for particular data-points. $95 \%$ confidence ellipses for each thyroid status group are also shown.

[Fig-2] exhibits plots of BMI versus age, and SBP:DBP ratio, MAP, and serum TSH and T4 levels versus BMI. Also shown are plots of SBP:DBP ratio versus BMI, serum T4 and serum TSH concentrations. The plot of BMI versus age revealed at least some distinction between the euthyroid and hyperthyroid groups [Fig-2](a), as did that of MAP versus BMI [Fig-2](b). However, the plot of serum TSH level versus BMI [Fig-2](C) shows a high level of discrimination between all three thyroid status classifications, whereas there was also a very clear separation visible between the hyperthyroid and both the euthyroid and hypothyroid groups (with also at least some 
distinction between these latter two groups) in the plot of serum T4 level versus BMI [Fig-2](d). Plots of SBP:DBP ratio versus BMI [Fig2](e) clearly showed that hyperthyroid patients had a very significantly higher SBP:DBP ratio and lower mean BMI values than those of both healthy control and hypothyroid patients. Moreover, particularly notable was that plots of SBP:DBP ratio versus serum T4 and TSH concentrations [Fig-2](f) and [Fig-2](g) respectively exhibited high levels of distinction between the thyroid status classifications.

Based on a BMI threshold cut-off of 23.0, we examined the frequencies of euthyroid and hypothyroid patients within each of the thyroid disease classification groups $(<23$ and those with values $\geq 23$ ) via a $X^{2}$ contingency table analysis, and found that there was a very strong association between these two classification criteria $[(p<0.0001$, Monte-Carlo simulation, [Fig-3](a), with a much higher proportion of hypothyroid patients in the elevated BMI group [odds ratio of $15.12(95 \% \mathrm{Cl}$ 9.33-24.50) and a likelihood ratio of 7.26]. Similarly, we also explored the association between this BMI classification and euthyroid vs. hyperthyroid status [Fig-3](b), and also found a significant association between these two criteria ( $p=$ 0.018), with a PETO odds ratio of 3.72 (95\% Cls 1.28-10.87).

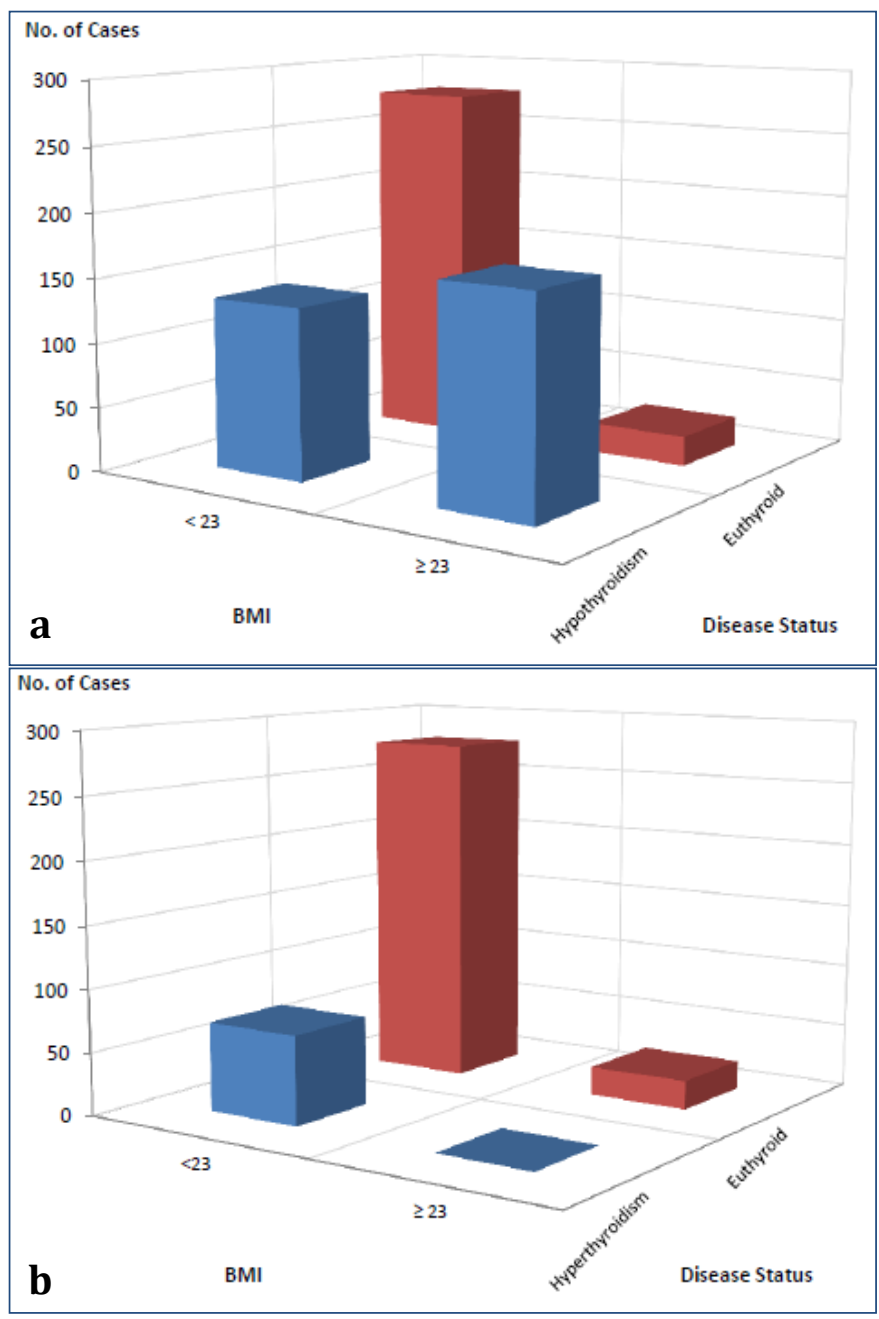

Fig. 3- 3D views of contingency table datasets demonstrating significant associations between BMI and Disease Status for comparisons of (a) hypothyroid with euthyroid patients $(n=300$ in each group, $p=0.0001)$, and (b) hyperthyroid with euthyroid patients $(n=$ 71 and 300 respectively, $p=0.018$ ). The $y$-axis represents the number of patients for each thyroid status criteria of classification.

\section{Multivariate Statistical Analysis \\ Partial Correlation Analysis}

We employed partial correlation analysis in order to explore similarities/dissimilarities between a MV dataset (consisting of patient serum T4 and TSH biomarker levels, BMl and age), and each of the four BP components (SBP, DBP, MAP and SBP:DBP ratio), the latter on an individual basis in view of interfering multicollinearity problems arising from the relatively high positive correlations existing between each of these BP parameters (either previously established or, for the SBP:DBP ratio index, discovered here). Partial correlation was chosen as an optimal method for the investigation of these relationships since it serves to provide realistic representations of relationships between the thyroid status and BP parameters in view of their independence of the influence of (i.e. co-correlations with) further, potentially interfering variables (unlike simple Pearson correlation coefficients)

On consideration of the clear 'between-thyroid status' differences in such relationships, we computed partial correlation coefficients [ $r_{b p}$ (n).th(1-4)] for each thyroid status classification [Table-2].

Table 2- Partial correlation coefficient $\left(r_{b p(n) . t h(1-4))}\right.$ values between individual BP components and blood serum TSH and T4 biomarker levels (IU/ml and $\mathrm{ng} / \mathrm{ml}$ respectively), age (yr.) and BMI (kg.m-2) parameters. The corresponding two-tailed significance $(p)$ values of these $r_{b p(n) . t h(1-4)}$ values are provided in brackets.

\begin{tabular}{|c|c|c|c|c|c|}
\hline $\begin{array}{l}\text { Thyroid Disease } \\
\text { Status }\end{array}$ & Parameter & SBP & DBP & MAP & SBP:DBP \\
\hline \multirow{4}{*}{ Hypothyroid } & TSH level & $\begin{array}{c}0.1359 \\
(0.0191)\end{array}$ & $\begin{array}{c}0.1864 \\
(0.00125)\end{array}$ & $\begin{array}{c}0.1745 \\
(0.00255)\end{array}$ & ns \\
\hline & T4 level & ns & ns & ns & ns \\
\hline & Age & $\begin{array}{c}0.1939 \\
(0.00078)\end{array}$ & $\begin{array}{c}0.2144 \\
(0.00020)\end{array}$ & $\begin{array}{c}0.2194 \\
(0.00014)\end{array}$ & \\
\hline & BMI & $\begin{array}{l}0.2589(< \\
0.00001)\end{array}$ & $\begin{array}{c}0.2156 \\
(0.00018)\end{array}$ & $\begin{array}{c}0.2528 \\
(0.000103)\end{array}$ & $\begin{array}{c}0.1193 \\
(0.0399)\end{array}$ \\
\hline \multirow{4}{*}{ Hyperthyroid } & TSH level & ns & ns & ns & ns \\
\hline & T4 level & ns & $\begin{array}{c}0.3498 \\
(0.0035)\end{array}$ & $\begin{array}{c}0.2906 \\
(0.0162)\end{array}$ & ns \\
\hline & Age & $\begin{array}{c}0.3382 \\
(0.0048)\end{array}$ & $\begin{array}{c}0.3931 \\
(0.00091)\end{array}$ & $\begin{array}{c}0.4078 \\
(0.00056)\end{array}$ & ns \\
\hline & BMI & ns & ns & ns & ns \\
\hline \multirow{4}{*}{ Euthyroid } & TSH level & ns & ns & ns & $\begin{array}{l}-0.1192 \\
(0.0401)\end{array}$ \\
\hline & T4 level & $\begin{array}{c}0.1432 \\
(0.0135)\end{array}$ & ns & $\begin{array}{c}0.1423 \\
(0.0140)\end{array}$ & ns \\
\hline & Age & ns & ns & ns & ns \\
\hline & BMI & ns & ns & ns & ns \\
\hline
\end{tabular}

ns: not significant

Although all the $r_{b p(n): t h(1-4)}$ values computed are relatively small, approximately one-third of them were statistically significant in view of the very large sample sizes employed in this investigation. Firstly, for the hypothyroid status group of patients, individual SBP, DBP and MAP values all significantly positively correlated with serum TSH concentration, BMI and age, whereas the SBP:DBP ratio index only correlated significantly and positively with BMI. Secondly, and in contrast, the hyperthyroid status classification exhibited strong positive correlations of the individual DBP and MAP BP components with both the serum T4 level $(p=0.0035$ and 0.0162 respectively) and age ( $p=0.00091$ and 0.00056 respectively) explanatory variables; the SBP component was only significantly positively cor- 
related with age, and no significant correlations of the SBP:DBP index to any of the explanatory variables were found. Thirdly, for the euthyroid (healthy control) group, significant positive correlations were found between the SBP, DBP and MAP components and serum $\mathrm{T} 4$ concentration, whereas, interestingly, a significant negative one was found between the SBP:DBP ratio parameter and serum TSH concentration. These results acquired are at least partially consistent with those acquired from the further MV analysis performed and described below.

\section{Permutation Tests via Redundancy Analysis (RDA)}

Primarily, we performed a series of permutation tests via RDA in order to explore relationships between BP components and thyroid disease status with its associated serum T4 and TSH biomarker level datasets. This test served to determine whether or not the thyroid status classifications of the study participants is significantly improved over that arising from any other random classification of these groups; the class labels of the healthy control, hypothyroid and hyperthyroid patients are permuted, and then randomly assigned to different patients. With these 'incorrect' disease class labels, a classification model was again computed; hence, the rationale was that with these 'incorrect' class labels, the computed model for classification purposes should be ineffective at class prediction (since the groups are generated randomly, the null hypothesis is that there are no differences between them). With repetition of this permutation test many times $(500,1,000$ or 2,000 times in this investigation), a null distribution of classifications which are expected to be insignificant were formed, and if the computed statistic (pseudo-F) lies outside at least the $95 \%$ or $99 \%$ confidence bounds of this distribution, then it could be concluded that there is a significant (linear) relationship between the two sets of parameters.

In this manner we utilised four experimental models: (1) a full RDA in which relationships between all BP components (SBP, DBP, SBP:DBP ratio and MAP values as response variables) to all thyroid status classifications (hyper and hypothyroid, and euthyroid) and biomarkers (serum T4 and TSH levels), together with BMI, age and gender as explanatory variables; (2) a partial RDA in which the conditioning effects of the $\mathrm{BMl}$, age and gender variables were removed from model (1); (3) a somewhat simpler full RDA model in which all of the above BP components served as response variables, and only the thyroid status classification was employed as the explanatory variable; and (4), as (3), but a partial RDA in which the conditioning effects of the BMI, age and gender variables were again removed.

Extremely highly significant relationships were found for all of the above four experimental models $(p<0.0001)$, results which clearly demonstrated powerful associations between thyroid disease status and each of the four BP components examined [Fig-4]. Indeed, for model (1), only the first and second factors significantly contributed to the total variance ( 80 and $20 \%$, respectively), and the order of each factorial contribution for the explanatory variables was euthyroid disease status $>$ BMI $>$ age $>$ serum TSH level $>$ hypothyroid disease status $>$ gender for factor 1 , and hyperthyroid disease status and serum T4 level for factor 2, i.e. the second component was ascribable to the hyperthyroid condition [these two factors represent linear combinations of (correlated) quantitative explanatory variables and are orthogonal (uncorrelated) to each other]. For the BP component (response) variables, however, contributions to factor 1 were MAP > SBP > DBP values, whereas, as expected, only the SBP:DBP ratio (highly significantly elevated in hyperthyroid patients over those in the hypothyroid and euthyroid classifications) contributed to the second factor.

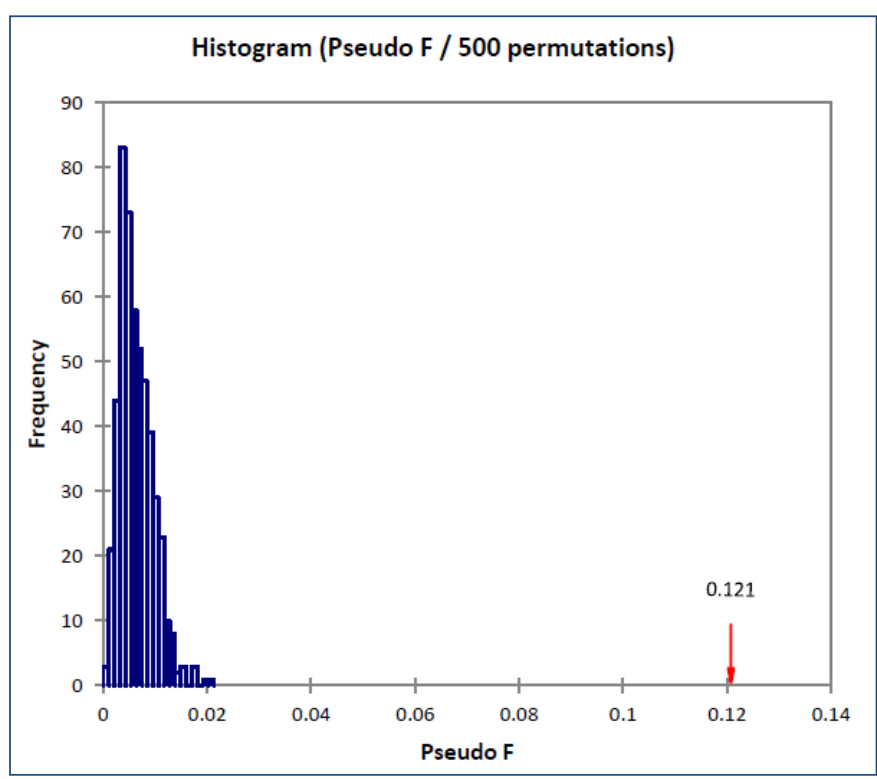

Fig. 4- Partial RDA permuation test demonstrating an extremely highly significant relationship between the blood pressure components SBP, DBP, SBP:DBP ratio and MAP values (response variables) and thyroid status (hypothyroid, euthyroid and hyperthyroid classifications), together with blood serum TSH and T4 biomarker levels (all serving as explanatory variables). In this model, the conditioning effects of the further explanatory variables gender, age and BMI were removed.

\section{Canonical Correlation Analysis (CCorA)}

-For this phase of the investigation, we elected to employ Canonical Correlation Analysis (CCorA) in order to investigate interrelationships between two series of datasets, the first (dependent) one comprising the clinical SBP, DBP, MAP, and SBP:DBP ratio BP indices, the second ('independent') one consisting of serum concentrations of the T4 and TSH thyroid biomarkers, an arbitrary disease score index (specifically, $-1,0$ and +1 for hypothyroid, euthyroid and hyperthyroid patient participants respectively), together with additional known BP-determining ones, i.e. age, BMl and gender, the latter expressed as a score of 0 for males and +1 for females. In view of multicollinearity problems within both these datasets, we primarily performed PCA in order to obtain the maximum possible number of orthogonal (uncorrelated) components from them; CCorA was then performed on the score vectors arising from these two sets of PCA components in order to evaluate the interrelationships between them, and also the dimensionality of such canonical correlations (these canonical dimensions represent latent variables which are themselves analogous to factors in FA, although such canonical variates serve to maximise the correlation between the two sets of variables). Both datasets were cubed roottransformed and then Pareto-scaled prior to PCA analysis.

The preliminary PCA step revealed that there were 3 major PCs isolated for the (independent, lateral $\mathrm{X}$ variable) thyroid biomarker level/disease score/age/BMl/gender score dataset, each with eigenvalues (mean number of predictor $X$ variables per $P C$ ) greater than or very close to 1 , the first (PC1) encompassing a linear combination of inversely-correlated serum T4 and TSH concentrations, and 
disease score index (eigenvalue 2.90 , with $48.3 \%$ of the variance explained), with loadings of $0.89,-0.92$ and 0.97 respectively, whilst the second (PC2) reflected a combination of positively-correlated age and BMI values (eigenvalue 1.28 , with $21.3 \%$ of the variance explained), with loadings of 0.81 and 0.61 respectively. However, the third PC (PC3) arose from gender score only (eigenvalue 0.93 , with $15.4 \%$ of variance explained) with a loading of 0.85 . The squared cosines of these variables' contributions towards their respective PCs were all greater than 0.50 , with the exception of that of BMl featured in PC2 (0.38). The Kaiser-Meyer-Olkin measure of sampling adequacy was $>0.50$ for all variables included in the model, and a corresponding PCA correlation plot is displayed in [Fig-5] (a).

However, for the (dependent) set of BP variables, prior PCA conducted was clearly segregated into 2 clearly-defined $P C s$, each with eigenvalues $>1$. The first (PC1) comprised a linear combination of the positively-correlated SBP, DBP and MAP parameters with loadings of $0.95,0.83$ and 0.99 respectively (eigenvalue 2.69, with $67.3 \%$ of variance explained), whilst the second (PC2) contained only the SBP:DBP ratio variable (which, as noted, appears to represent a clear hypertensive diagnostic criterion for hyperthyroid patients), with a strong PC2 loading of 0.94 (eigenvalue 1.30, with $32.6 \%$ of variance explained). The squared cosines of these variables' contributions towards their respective PCs ranged from 0.69 to 0.97 , and a PCA correlation plot for this dataset is exhibited in [Fig5](b).

Subsequently, we applied a CCorA model in order to seek and fur- ther evaluate canonical variates (and their dimensionality) between the orthogonal (dependent variate) BP PC score vectors (specifically, the first comprising a linear combination of SBP, DBP and MAP parameters, the second SBP:DBP ratio alone), and those arising from the (independent) thyroid disease biomarkers/disease score/further latent variables, specifically, age, BMI and gender score (for the latter variable dataset, the 3 PCs described above were incorporated into the model). Performance of this technique revealed that there were 2 canonical dimensions between the two sets of PCA component score vectors, the first of which was found to be very highly significant ( $p<0.0001$, Wilks'-Lambda test); these first and second dimensions corresponded to canonical correlations of 0.308 and 0.190 respectively and accounted for 72.4 and $27.6 \%$ of the total variance.

[Fig-5](c) shows a canonical correlation plot of the second versus the first 'factor of factors' arising from this form of CCorA. This analysis clearly revealed that the first thyroid PC (containing positivelyloading serum T4 level and disease score values, and negativelyloading serum TSH concentration), contributed to the first CCorA dimension in an extremely significant manner, and was strongly and positively related to the second $\mathrm{BP} P C$, i.e. that consisting of the $\mathrm{SBP}: \mathrm{DBP}$ ratio index alone. Hence, as expected from the statistical analyses performed, elevated serum T4 and diminished serum TSH concentrations, which serve as biomarkers which are characteristic of hyperthyroid patients (which has a disease score of +1 in this model system), are unequivocally correlated to the unique SBP:DBP parameter developed in this investigation.
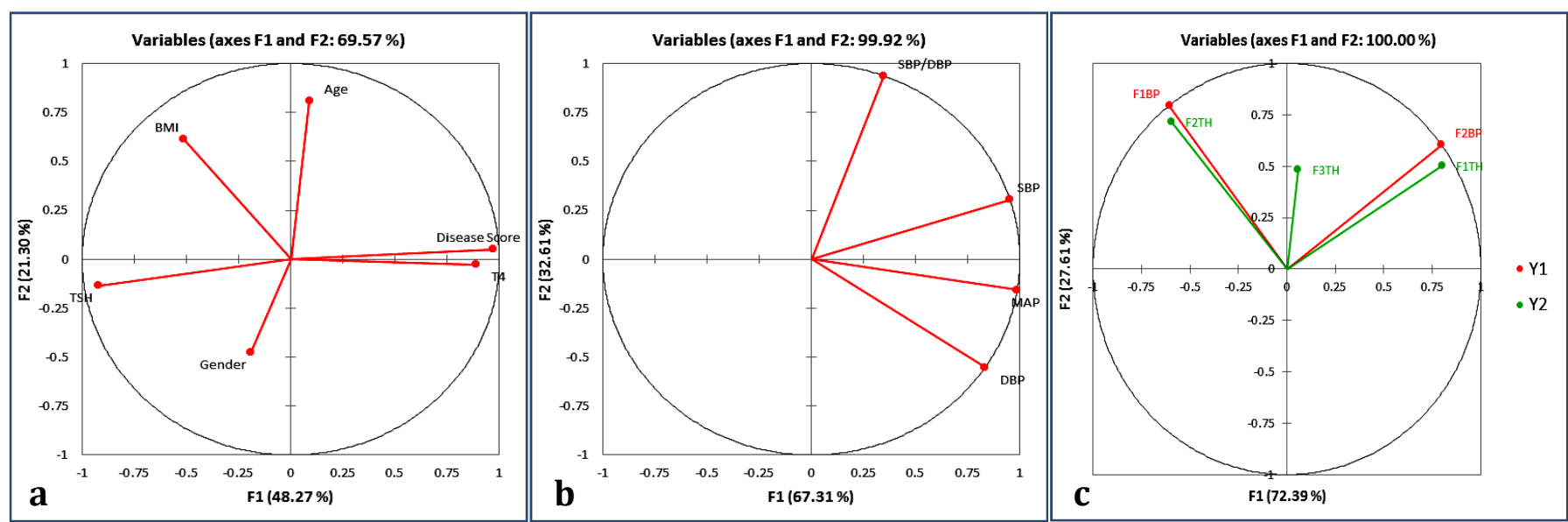

Fig. 5- (a) Primary Principal Component Analysis (PCA) performed on the 'dependent' BP Y variable dataset consisting of SBP, DBP, MAP and SBP:DBP ratio indices; (b) as (a), but for the 'independent' $X$ variable dataset comprising thyroid biomarkers (blood serum T4 and TSH levels), arbitrary thyroid disease score (-1 for hypothyroid, 0 for euthyroid and +1 for hyperthyroid patients), age, BMl and gender score $(0$ and +1 for males and females respectively); (c) plot of factor 2 (F2) versus factor 1 (F1) for canonical correlation analysis (CCorA) of orthogonal principal components (PCs) obtained via the prior PCA analysis of the blood pressure parameter dataset $(\mathrm{Y})$ consisting of SBP, DBP and MAP values (responsible for PC1) and the diagnostic SBP:DBP ratio (the only significant contributing variable to $\mathrm{PC} 2$ ), and the second dataset (X) comprising thyroid disease biomarkers (blood serum T4 and TSH levels) and disease score (responsible for PC1), together with participant age and BMl parameters (responsible for PC2) and gender score (accounting for PC3 alone). Canonical correlations (i.e., standardised canonical coefficients) between the first BP dataset input PC score vectors were -0.606 (first BP PC) and 0.796 (second BP PC) for F1, and 0.796 (first BP PC) and 0.606 (second BP PC) for F2. Canonical correlations between the second dataset input variables (3 orthogonal PC score vectors arising from linear combinations of blood serum thyroid disease biomarkers and the thyroid disease score, age and BMI values, and gender score) were 0.800 (first lateral variable PC), -0.597 (second lateral variable PC), and 0.057 (third lateral variable PC) for $\mathrm{F} 1$, and 0.502 (first lateral variable PC), 0.717 (second lateral variable $\mathrm{PC}$ ) and 0.483 (third lateral variable PC) for F2. The complete thyroid status dataset was analysed in the example shown. Both datasets were cubed root-transformed and then Pareto-scaled prior to performance of the primary PCA analysis.

Also notable is the inter-relationship between the first BP dataset PC (i.e. that incorporating the strongly- and positively-correlated
SBP, DBP and MAP indices) and the second thyroid disease variable one (that consisting of the age and BMI predictors), an observa- 
tion which is expected in view of the known correlations between the variables featured in each of the two sets of orthogonal PCs isolated in this CCorA model. The third (gender status) PC developed from the 'independent' $X$ variable dataset was found not to be significantly related to either of the BP dataset PCs [Fig-3](c).

\section{Principal Component Analysis (PCA)}

PCA analysis of a (simplified) dataset, which included only the BP components as predictor variables, and only thyroid status as a (qualitative) supplementary one, revealed at least some 'clusterings' of the these thyroid status classifications, the differences observed between the hyperthyroid and healthy control groups being especially notable ([Fig-6] shows a plot of principal component 2 (PC2) versus principal component 1 (PC1) for the varimax-rotated dataset). As noted for the RDA and CCorA performed above, for this form of MV analysis, SBP, DBP and MAP values were significant contributors to PC1, whereas only the SBP:DBP ratio significantly contributed to PC2. PC1 and PC2 accounted for 67.9 and $31.9 \%$ of the total variance respectively $(99.8 \%$ in total). For this analysis, Bartlett's sphericity test was extremely statistically significant $(p<10$ ${ }^{12}$ ), and squared cosine values for the BP index variables were 0.91 (SBP), 0.72 (DBP), 0.98 (MAP) and 0.89 (SBP:DBP ratio).

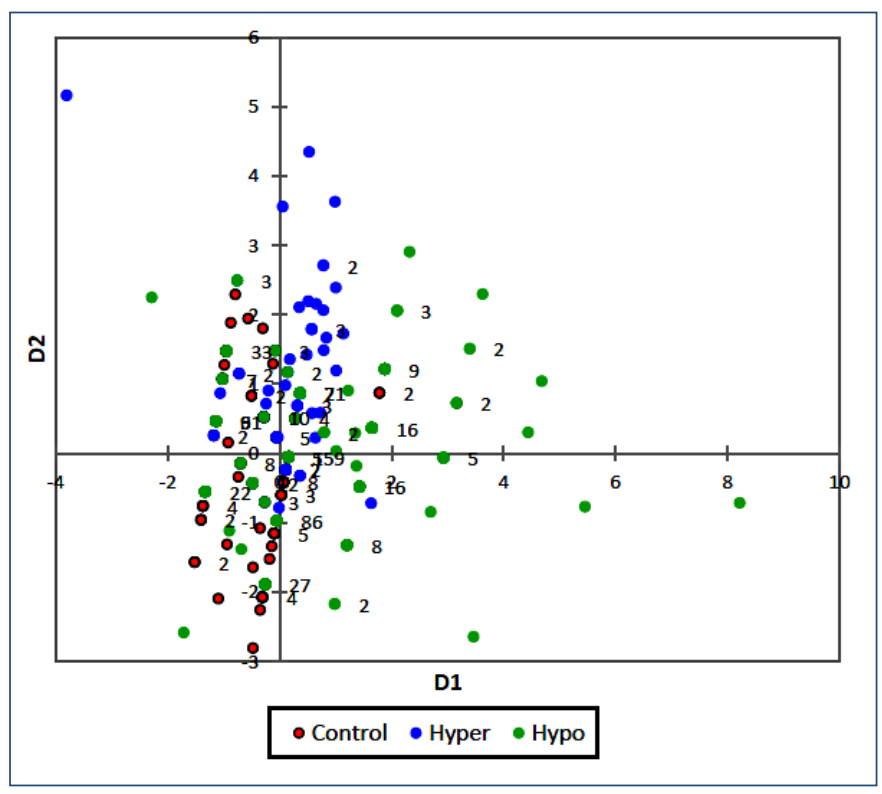

Fig. 6- Scores plot of principal component 2 versus principal component 1 (D2 versus D1) for a principal component analysis (PCA) model which only incorporated blood pressure variables (SBP, DBP, SBP:DBP ratio and MAP indices) as explanatory (predictor) variables of thyroid status [which represented the only supplementary variable (i.e., as a qualitative one)]. For this analysis, a rotation matrix was first applied to the component co-ordinate matrix, and a Kaiser normalisation was performed during the rotation calculation. The non-scalar numbers indicate frequencies $>1$ for particular datapoints. Factor loadings after varimax rotation were 0.798 (SBP), 0.977 (DBP), 0.980 (MAP) and -0.006 (SBP:DBP ratio) for D1, and 0.600 (SBP), -0.211 (DBP), 0.195 (MAP) and 0.999 (SBP:DBP ratio) for $\mathrm{D} 2$. Each $\mathrm{BP}$ variable was standardised prior to performing this PCA.

\section{Discussion}

Univariate statistical analyses revealed extremely highly significant 'between-thyroid status' differences $(p<0.0001)$ for the SBP, DBP,
MAP, and uniquely SBP:DBP ratio parameters. Moreover, BMI was significantly elevated in hypothyroid status patients over those of the healthy control and hyperthyroid status groups $(p<0.0001)$, and healthy controls had a significantly greater mean BMI value than that of hyperthyroid patients $(p<0.0001) \cdot X^{2}$ contingency table analysis revealed very strong associations between overweight status (BMl<or $\geq$ a threshold value of 23) and (1) euthyroid and hypothyroid disease status classifications $(p<0.0001)$ and $(2)$ euthyroid and hyperthyroid classifications $(p<0.018)$. Partial correlation analysis demonstrated significant or highly significant correlations of (1) SBP, DBP and MAP BP components with blood serum TSH level for the hypothyroid group (all positive); DBP and MAP values with serum T4 concentration for the hyperthyroid group (also both positive); and intriguingly, SBP, DBP and MAP indices with serum T4 level (all positive), plus a negative one between SBP:DBP ratio and serum TSH concentrations for the euthyroid group. These results clearly indicate the importance and significance of systolic and/or diastolic hypertension in hypo and hyperthyroidism.

MV permutation tests performed via RDA demonstrated powerful associations between thyroid disease status and each of the $\mathrm{BP}$ components. Moreover, our CCorA model for the analysis of PCs derived independently from (1) the dependent BP index $Y$ variable, and (2) the thyroid biomarker and score, age, BMI and age score lateral potential predictor $X$ variable datasets revealed two significant dimensions between the two BP PCs and the three arising from the correlated predictor variables. The first of these encompassed powerful positive correlations between a factor with only the SBP:DBP ratio parameter as a significant contributory variable, and that comprising the two serum thyroid biomarker concentrations and disease score (specifically, with positively-correlated thyroxine concentration and disease score, but negatively-correlated TSH level), and hence these results confirm that the newly-developed SBP:DBP ratio index significantly increases with increasing increments of serum T4 level, but diminishes with that of TSH (of course, this ratio was also highly positively correlated with disease score in view of the extremely elevated magnitude of this parameter in hyperthyroid patients over and above those of the hypo- and euthyroid classifications). Moreover, as expected, the second dimension reflected a positive (albeit less significant) association between the PC comprising the highly-correlated SBP, DBP and MAP indices, and that arising from a linear combination of the age and BMI variables in the predictor variable dataset. These results are consistent with those acquired from the above RDA conducted. Independent PCA scores plots revealed at least some clustering of the three thyroid status classifications, and, as observed with CCorA model performed, the SBP, DBP and MAP values were significant contributors to the first PC, whereas only the SBP:DBP ratio significantly contributed to the second.

Although essential hypertension is more common, secondary hypertension is increasing and is now prevalent in view of associated disorders, which are more common in females [12]. Furthermore, thyroid disorder is the most common endocrinological disorder, and hypertension is more often observed in thyroid diseases. However, to date there are only limited studies confirming this association [3]. Isolated systolic hypertension is common in hyperthyroidism, and systo-diastolic hypertension (with an associated elevated DBP rather than SBP values) is prevalent amongst hypothyroid patients [3, 4]. Hence, the SBP and SBP:DBP ratio components are expected to be greater in hyperthyroid subjects. Although both SBP and DBP values are elevated in hypothyroidism, the SBP:DBP ratio will be 
expected to be lower in patients with this condition, as observed here. Moreover, since the thyroid status-mediated increase in DBP is greater than that of SBP in hypothyroidism, MAP values are also expected to be higher in this class of patients.

\section{Mechanisms of Hypertension in Hyperthyroidism}

In hyperthyroidism, serum T3 and T4 levels are elevated, and its TSH concentration is diminished. T3, the active form of the thyroid hormone system, reduces systemic vascular resistance through dilatation of the arteriole and relaxation of vascular smooth muscle. Hyperthyroidism increases heart rate (HR), cardiac contractility and blood volume, and HR is a strong predictor of $\mathrm{CO}$ since it affects both systolic and diastolic function [13]. In hyperthyroidism, HR may be elevated up to $40 \%$ of its basal value, and CO increases up to $300 \%$ when compared with that of hypothyroid patients [14]. SVR largely regulates afterload which, in turn, depends on the arterial compliance and aortic impedance. Moreover, an increased capillary density and expression of vascular endothelial growth factors have been reported in thyroid diseases $[15,16]$.

Thyroid hormone-induced vascular muscle relaxation through an increased level of NO production from endothelial cells has been demonstrated in experimental models [16]. T3 increases the synthesis of cardiac proteins associated with cardiac hypertrophy through the activation of myocyte-specific genes (9). Furthermore, the non-genomic effect of T3 includes its effect on membrane ion channels for sodium, potassium, and calcium, actin polymerisation, mitochondrial proteins and further intracellular cardiac signaling pathways [15]. The above cardiogenic mechanisms explain the pathogenesis of hypertension in hyperthyroidism. However, antihypertensive treatment with ACE inhibitors in experimental models reduces $B P$, but not $C O$ and $H R[16,17]$, suggesting the possibility that some other non-cardiogenic mechanisms contribute to hypertension in thyroid disorders. Even in a hypertensive euthyroid population, thyroid hormones may be associated with left ventricular (LV) remodelling and mass, independent of LV and renal dysfunctions [18].

The juxtaglomerular apparatus of the kidney is sensitive to volume and BP. Since T3 decreases SVR and afterload, there will be a decrease in mean BP, which, in turn, activates the RAAS cascade and promotes an elevation in renin synthesis, blood volume and preload that contributes to $\mathrm{CO}$ value, which is raised in hyperthyroidism [19]. An increased number of $\beta$-adrenergic receptors are found in the renal cortex of experimental models with hyperthyroidism, and $\beta$-adrenergic stimulation enhances renin secretion [17]. Additionally, thyroid hormones influence baroreflex function and the autonomic nervous system, which are essential for the maintenance of both HR and BP, and hence both play a significant role in the development and progression of hypertension.

In view of the above hemodynamic effects, increases and decreases in the SBP and DBP components, respectively, will be expected, and consequently pulse pressure will be elevated. Pulse pressure serves as a marker of increased arterial stiffness, and also serves as a high index factor for cardiovascular risk, especially in elderly populations [20]. In a previous study, a reduction in nocturnal BP was identified amongst hyperthyroid subjects with hypertension [21]. An additional investigation involving a 24-hr. BP monitor demonstrated a similar mean value of this parameter in normotensive hyperthyroid patients when compared to that of normotensive euthyroid individuals [22]. Treatment of hyperthyroidism decreases pulse pressure, HR and $\mathrm{CO}$, and subsequently BP. In a further report, hypertension was found to normalise to baseline normal range values following thyroidectomy for functional thyroid gland adenocarcinoma [23].

Sub-clinical hyperthyroidism (SHY) is characterised by normal serum T3 and T4 levels coupled with diminished TSH ones, and this condition is also common. However, predominantly, SHY never produces any clinical signs. Interestingly, Tamer et.al. noted an increased left ventricular (LV) mass and cardiac workload in SHY patients [24]. However, to date there is no evidence available for hypertension in SHY patients, which has been confirmed by a community-based study [25].

\section{Mechanisms of Hypertension in Hypothyroidism}

Hypothyroidism is the second most common cause for secondary hypertension (following renal hypertension); however, it is often overlooked. The prevalence of hypertension amongst hypothyroid patients varies from 1 to $50 \%$ [26]. Such a substantial variation in prevalence is ascribable to variable criteria in defining hypothyroid disease and hypertension, and also to differences in the age of subjects studied [27]. As discussed above, thyroid hormones play a key role in the modulation of hemodynamic parameters. Hypothyroidism increases SVR and arterial stiffness in view of its vasoconstrictor effect on vascular smooth muscle [28], sympathetic nervous system stimulation, and decreases in $\beta$-adrenergic receptors (coupled with a parallel increase in a-adrenergic response) [29]. Hypothyroidism causes myxedema of the arterial wall which, in turn, gives rise to hypertension [28].

Hypothyroidism reduces renal blood flow and the glomerular filtration rate (GFR), with inappropriate $A D H$ secretion (i.e., a mildly increased $A D H)$. Consequently, there is free water retention with an associated increase in total body water, a decrease in intravascular volume and hyponatremia $[30,31]$. Sodium transport in vascular smooth muscle and the kidney is regulated by the lithium-sodium $\left(\mathrm{Li}^{+}-\mathrm{Na}^{+}\right)$counter transport mechanism; indeed, in essential hypertension, there is an increased $\mathrm{Li}^{+}-\mathrm{Na}^{+}$counter transport [32]. Thyroid hormones may also alter $\mathrm{Li}^{+}-\mathrm{Na}^{+}$transport, which is more pronounced in hypothyroidism, and hence hypothyroidism may cause hypertension in this manner [33]. In addition, hypothyroidism gives rise to low renin and angiotensin levels, which can cause saltsensitive hypertension $[34,35]$. Hypothyroidism is frequently associated with metabolic syndrome and obesity $[10,36]$, which are also predisposing factors for hypertension. Elevated plasma TSH concentrations (probable hypothyroidism) in patients with metabolic syndrome independent of serum T3 and T4 biomarker values suggest it to be a component of the metabolic syndrome [37]. Asvold, et al found a linear increase in both systolic and diastolic BP components with increasing serum TSH levels in both men and women (i.e. significant positive correlations were observed) [5]. In a further study, mean day and night time DBP values were also found to increase linearly with TSH levels [38]. Intriguingly, diastolic BP elevated significantly during changes from hyperthyroidism to hypothyroidism, and also normalised to normotensive values after the achievement of euthyroid status and low plasma renin levels in hypertensive hypothyroidism [39].

Sub-clinical hypothyroidism (SHO) is classified as a condition associated with high serum TSH, but normal serum T3 and T4 concentrations, with or without clinical symptoms; however, this condition has been associated with diastolic hypertension [25]. Various stud- 
ies have indicated an association of hypertension with $\mathrm{SHO}$ $[7,25,40]$, whereas Duan, et al [8] did not find any such association. Notwithstanding, Dagre, et al [41] found a strong relationship between $\mathrm{SHO}$ and both systolic and diastolic classes of hypertension. Moreover, endothelial dysfunction is more prevalent in hypothyroid patients [42], and pregnant women with sub-clinical hypothyroidism have an increased risk for severe pre-eclampsia when compared with euthyroid subjects [43].

In one study [44], an elevated serum TSH concentration was noted in families with a high prevalence of hypertension, which suggests the possibility of genetic polymorphism of this biomarker with type-2 iodothyroninedeiodinase genes which are involved in the modulation of BP regulation and serum levels of this biomarker. In hypothyroid patients, treatment with $\mathrm{T} 4$ reduces arterial stiffness, vascular resistance and BP [41]. Hence, early diagnosis and management are required in such patients in order to reduce cardiovascular risk status.

\section{Treatment of Hypertension in Thyroid Disorders}

In thyroid disorders, hypertension is reversible once thyroid function attains normalisation status, although this may not necessarily occur shortly thereafter. However, treatment with antihypertensive drugs is mandatory in the case of moderate to severe hypertension. Isolated systolic hypertension represents the strongest risk factor for cardiovascular disease, which is common in hyperthyroidism [3]. $\beta$-adrenergic blockers are preferred in such cases, since they not only reduce $\mathrm{BP}$, but also alleviate further symptoms of hyperthyroidism such as tremor, tachycardia and anxiety [44]. Propranolol inhibits the peripheral conversion of $\mathrm{T} 4$ to $\mathrm{T} 3$, a more active form of these thyroid hormones. Individuals with relative contra-indications for $\beta$-adrenergic blockers may tolerate b1-selective drugs more effectively. Calcium channel blockers (CCBs) may be considered if patients are unable tolerate $\beta$-blockers, and felodipine has been shown to reduce BP and SVR in hypertensive hypothyroid patients who did not respond to hormone replacement [45]. ACE inhibitors offer a potential benefit to hypertensives with thyroid disorders, since they reduce intraglomerular pressure [19]. In most cases, hypertension associated with hypothyroidism is salt-sensitive, although in hyperthyroidism it is salt-resistant. Hence, a typical saltsensitive and low renin form of hypertension is frequent in hypothryroidism, and this is more effectively treated with a low sodium diet, CCBs and a diuretic.

\section{Conclusions}

Isolated systolic hypertension and an increased pulse pressure are more common in hyperthyroidism, whereas combined systodiastolic hypertension (in which elevations in DBP exceed those in SBP) is more pronounced in hypothyroidism. We have also noted significant associations between SBP:DBP ratios, MAP values, and thyroid disease status. Indeed, employment of the SBP:DBP ratio may serve as a valuable diagnostic index for monitoring the development of hypertension and its response to treatment in hyperthyroid patients. Therefore, practitioners may be motivated to record $\mathrm{BP}$ in thyroid disorders and provide appropriate medications in order to reduce cardiovascular mortality/morbidity risks. The use of 24 hr. ambulatory BP measurements will not only facilitate this diagnosis, but also provide valuable information regarding load, circadian variation and BP variability, which are all considered as important risk factors and surrogate markers for cerebro and cardiovascular events.

\section{Limitations}

The limitations of this investigation are that correlations of the dataset acquired with duration of illness were not explored, $24 \mathrm{hr}$. ABP was not utilised, there was not a follow-up of BP in these patients subsequent to the initiaton of specific thyroid disease treatment(s), and the non-estimation of serum T3 levels (each of these are ascribable to resource limitations). Hence, a larger clinical trial with a long-term follow-up period is warranted.

\section{Summary}

\section{What is known about the topic?}

Isolated systolic hypertension and an increased pulse pressure are more common in hyperthyroidism, whereas combined systodiastolic hypertension (in which elevations in DBP exceed those in $\mathrm{SBP}$ ) is more pronounced in hypothyroidism.

The use of $24 \mathrm{hr}$. ambulatory BP measurements will not only facilitate this diagnosis, but also provide valuable information regarding load, circadian variation and BP variability, which are all considered as important risk factors and surrogate markers for cerebro- and cardiovascular events.

\section{What this study adds on?}

We have also noted a significant association between SBP:DBP ratios, MAP values, and thyroid disease status.

Indeed, employment of the SBP:DBP ratio may serve as a valuable diagnostic index for monitoring the development of hypertension and its response to treatment in hyperthyroid patients.

Therefore, practitioners may be motivated to record BP in thyroid disorders and provide appropriate medications in order to reduce cardiovascular mortality/morbidity risks.

Authors Contributions: RM: worked on the questionnaire, study design, ethical committee application and approval procedure, data collection and drafting of the manuscript; MG: study design, data interpretation, statistical analysis, drafting of the manuscript and revision; RN: study design, data collection; EN: study design, data collection; LJ: ethical committee application and approval procedure, supervision of work and data collection; PT: study design, questionnaire, ethical committee application and approval procedure, work supervision and manuscript revision.

Acknowledgements: We would like to acknowledge and thank, the Ethical committee and Dean of Chennai Medical College Hospital and Research Centre, India, for their valuable support in the completion of this project.

Funding: There was no finanical interest for this study and we have not obtained any funds for the completion of project including manuscript preparation.

Competing Interests: All authors declare that they have no competing interests.

\section{References}

[1] Abdu T.A., Elhadd T., Pfeifer M. \& Clayton R.N. (2001) Trends in Endocrinology \& Metabolism, 12(6), 257-265.

[2] Cappola A.R. \& Ladenson P.W. (2003) The Journal of Clinical Endocrinology and Metabolism, 88(6), 2438-2444.

[3] Prisant L.M., Gujral J.S., Mulloy A.L. (2006) Journal of Clinical Hypertension, 8(8), 596-599. 
[4] Mazza A., Beltramello G., Armigliato M., Montemurro D., Zorzan S., Zuin M., Rampin L., Marzola M.C., Grassetto G., Al-Nahhas A. \& Rubello D. (2011) Annales d'endocrinologie, 72(4), 296303.

[5] Åsvold B.O., Bjøro T., Nilsen T.I. \& Vatten L.J. (2007) The Journal of Clinical Endocrinology and Metabolism, 92(3), 841-845.

[6] Åsvold B.O., Bjøro T. \& Vatten L.J. (2013) European Journal of Endocrinology, 169(1), 73-82.

[7] Takashima N., Niwa Y., Mannami T., Tomoike H., \& Iwai N. (2007) Circulation Journal, 71(2), 191-195.

[8] Duan Y., Peng W., Wang X., Tang W., Liu X., Xu S., Mao X., Feng S., Feng Y., Qin Y., Xu K., Liu C. \& Liu C. (2009) Endocrine, 35(2), 136-142.

[9] Klein I. \& Danzi S. (2007) Circulation, 116(15), 1725-1735.

[10]Kota S.K., Meher L.K., Krishna S.V.S. \& Modi K.D. (2012) Indian Journal of Endocrinology and Metabolism, 16(2), S332S333.

[11]Consultation WHOE (2004) Lancet, 363(9403), 157-163.

[12]Tagle V.R., Acevedo M. \& Valdes G. (2013) Revista Medica de Chile, 141(2), 237-247.

[13]Klein I. \& Ojamaa K. (2001) New England Journal of Medicine, 344(7), 501-509.

[14]Kaptein E.M., Sanchez A., Beale E. \& Chan L.S. (2010) The Journal of Clinical Endocrinology \& Metabolism, 95(10), 45264534.

[15]lordanidou A., Hadzopoulou-Cladaras M. \& Lazou A. (2010) Molecular and Cellular Biochemistry, 340(1-2), 291-300.

[16]Rodriguez-Gomez I., Banegas I., Wangensteen R., Quesada A., Jimenez R., Gomez-Morales M., O'Valle F., Duarte J. \& Vargas F. (2013) The Journal of Endocrinology, 216(1), 43-51.

[17]Haro J.M., Sabio J.M. \& Vargas F. (1992) Journal of Endocrinological Investigation, 15(8), 605-608.

[18]lida M., Yamamoto M., Ishiguro Y., Yamazaki M., Honjo H., Kamiya K. (2012) Journal of the American Society of Hypertension, 6(4), 261-269.

[19]Ganong W.F. (1982) Life Sciences, 30(7-8), 561-569.

[20]Palmieri E.A., Fazio S., Palmieri V., Lombardi G. \& Biondi B. (2004) European Journal of Endocrinology, 150(6), 757-762.

[21]Schrader J. \& Middeke M. (1994) British Medical Journal, 308 (6929), 630-632.

[22]Kohno I., Iwasaki H., Okutani M., Mochizuki Y., Sano S., Satoh Y., Ishihara T., Ishii H., ljiri H., Komori S. \& Tamura K. (1998) Chronobiology International, 15(4), 337-347.

[23]Simpson A.C. \& McCown J.L. (2009) Journal of the American Veterinary Medical Association, 235(12), 1474-1479.

[24]Tamer I., Sargin M., Sargin H., Seker M., Babalik E., Tekce M., \& Yayla A. (2005) Endocrine Journal, 52(4), 421-425.

[25]Walsh J.P., Bremner A.P., Bulsara M.K., O'Leary P., Leedman, P.J., Feddema P. \& Michelangeli V. (2006) Clinical Endocrinology, 65(4), 486-491.

[26]Vanderpump M.P. \& Tunbridge W.M.G. (2002) Thyroid, 12(10), 839-847.

[27] Graettinger J.S., Muenster J.J., Checchia C.S., Grissom R.L. \& Campbell J.A. (1958) Journal of Clinical Investigation, 37(4),
502-510.

[28]Obuobie K., Smith J., Evans L.M., John R., Davies J.S. \& Lazarus J.H. (2002) The Journal of Clinical Endocrinology \& Metabolism, 87(10), 4662-4666.

[29]Saito I. \& Saruta T. (1994) Endocrinology and Metabolism Clinics of North America, 23(2), 379-386.

[30]Montenegro J., González O., Saracho R., Aguirre R., González Ó. \& Martínez I. (1996) American Journal of Kidney Diseases, 27(2), 195-198.

[31]Allon M., Harrow A., Pasque C.B. \& Rodriguez M. (1990) Journal of the American Society of Nephrology, 1(2), 205-210.

[32]Canessa M., Adragna N., Solomon H.S., Connolly T.M. \& Tosteson D.C. (1980) New England Journal of Medicine, 302(14), 772-776.

[33]Brent G.A., Canessa M., \& Dluhy R.G. (1989) The Journal of Clinical Endocrinology \& Metabolism, 68(2), 322-328.

[34]Dzau V.J. \& Herrmann H.C. (1982) Life Sciences, 30(7-8), 577584.

[35]Marcisz C., Jonderko G. \& Kucharz E.J. (2001) American Journal of Hypertension, 14(10), 995-1002.

[36]Nakajima Y., Yamada M., Akuzawa M., Ishii S., Masamura Y., Satoh T., Hashimoto K., Negishi M., Shimomura Y., Kobayashi I., Andou Y. \& Mori M. (2013) The Journal of Clinical Endocrinology and Metabolism, 98(8), 3280-3287.

[37]Chugh K., Goyal S., Shankar V. \& Chugh S.N. (2012) Indian Journal of Endocrinology and Metabolism, 16(6), 958-961.

[38]Turchi F., Ronconi V., di Tizio V., Boscaro M. \& Giacchetti G. (2011) American Journal of Hypertension, 24(12), 1274-1279.

[39]Streeten D.H., Anderson G.H.Jr., Howland T., Chiang R., Smulyan H. (1988) Hypertension, 11(1), 78-83.

[40]Volzke H., Ittermann T., Schmidt C.O., Dorr M., John U., Wallaschofski H., Stricker B.H., Felix S.B. \& Rettig R. (2009) European Journal of Endocrinology, 161(4), 615-621.

[41]Dagre A.G., Lekakis J.P., Papaioannou T.G., Papamichael C.M., Koutras D.A., Stamatelopoulos S.F. \& Alevizaki M. (2005) International Journal of Cardiology, 103(1), 1-6.

[42]Taddei S., Caraccio N., Virdis A., Dardano A., Versari D., Ghiadoni L., Salvetti A., Ferrannini E. \& Monzani F. (2003) The Journal of Clinical Endocrinology and Metabolism, 88(8), 37313737.

[43]Wilson K.L., Casey B.M., McIntire D.D., Halvorson L.M. \& Cunningham F.G. (2012). Obstetrics and Gynecology, 119(2-1), 315 -320 .

[44]Trepanier L.A. (2006) Clinical Techniques in Small Animal Practice, 21(1), 22-28.

[45]Dernellis J. \& Panaretou M. (2002) American Heart Journal, 143 (4), 718-724. 